\title{
Immunomodulatory treatment with thalidomide in experimental leptospirosis in Golden Syrian hamsters (Mesocricetus auratus)
}

\author{
Luciane Marieta Soares $^{a, b}$, Julio Oliveira Macedo ${ }^{a, b}$, Everton Cruz de Azevedo ${ }^{b}$, Cleiton Silva Santos ${ }^{a}$, Marina de

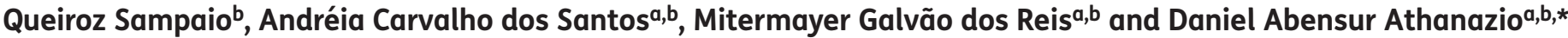

\author{
${ }^{a}$ Centro de Pesquisa Gonçalo Moniz, Fundação Oswaldo Cruz, Salvador, BA, 40296-710, Brazil; ' Universidade Federal da Bahia, \\ Salvador, BA, 40025010, Brazil
}

*Corresponding author: Present address: Universidade Federal da Bahia, Faculdade de Medicina da Bahia, Departamento de Patologia e Medicina Legal, Praça XV de novembro, s/n - Largo do Terreiro de Jesus, 40025010 - Salvador, BA-Brazil; Tel: +55 7132835563 ext. 41

Received 15 August 2013; revised 11 November 2013; accepted 15 November 2013

\begin{abstract}
Background: The benefit of antibiotics in leptospirosis is limited when treatment is started four days after symptoms appear, and new adjuvant therapeutic options are urgently needed.
\end{abstract}

\begin{abstract}
Methods: Hamsters (Mesocricetus auratus) were infected by Leptospira interrogans strain L1-130, and groups were assigned based on no treatment (NONE), thalidomide only (TAL), ampicillin only (AMP) or both (AMPTAL). Treatment was started two days after the onset of symptoms (experiment 1 ) and immediately after detection of the first death (experiment 2).

Results: Experiment 1: all hamsters from the groups AMP and AMP-TAL survived ( $n=8)$, while all hamsters from groups NONE $(n=6)$ and TAL $(n=8)$ died. The AMP and the AMP-TAL groups showed no renal or liver pathology and absent or very low leptospiral burden in target organs. Experiment 2: lethal outcome was observed in 6/6 hamsters in the NONE group, 8/8 in the TAL group, and 6/8 in both the AMP and AMP-TAL groups. Thalidomide showed no survival benefit when compared to hamsters treated with ampicillin alone. The TAL, AMP and AMP-TAL groups had very low tissue leptospiral counts.
\end{abstract}

Conclusion: Thalidomide had minimal impact on survival in the late treatment of leptospirosis hamster model.

Keywords: Animal disease models, Leptospira, Leptospirosis, Mesocricetus auratus, Thalidomide

\section{Introduction}

Leptospirosis is a widespread zoonosis caused by pathogenic leptospires. The disease occurs in distinct settings such as rural and/ or occupational endemics, large urban areas with poor sanitation, or water sports and ecotourism related exposure. ${ }^{1}$ Most human infections are asymptomatic or cause mild febrile illness that is indistinguishable from diseases caused by other infectious agents. However, $5-10 \%$ of human infections will evolve to severe forms, such as the Weil's triad of acute renal failure, hemorrhages and jaundice (with 5-30\% related case fatality) and severe pulmonary hemorrhagic syndrome (with $\geq 50 \%$ related case fatality). ${ }^{2-4}$

Additional tools for the treatment of severe forms are urgently needed because a major issue in the management of patients is the limited effect of antibiotics when started more than four days after clinical onset. ${ }^{4}$ The hamster model reproduces target organ pathology of human severe leptospirosis including jaundice, tubulointerstitial nephritis, liver cell disarray and pulmonary hemorrhages. ${ }^{5}$ In experimental leptospirosis, ampicillin is the most popular and standardized antibiotic treatment used by different groups in therapeutic assays. ${ }^{6-9}$ We have previously developed a hamster model of experimental leptospirosis to reproduce the late start of antibiotic (ampicillin) therapy and to test the adjuvant effect of the antioxidant $\mathrm{N}$-acetylcysteine ${ }^{7}$ and methylene blue-an inhibitor of soluble guanylyl cyclase-downstream the action of nitric oxide synthases ${ }^{6}$ which had no additional benefit in experimental disease. Another potential target for adjuvant therapy in leptospirosis may be the cytokine cascade related to tumor necrosis factor alpha (TNF- $\alpha$ ) because leptospirosis seems to share the association of high levels of pro-inflammatory markers of severity and lethality with some forms of sepsis, such as meningococcemia. ${ }^{10-12}$ In addition, most data suggest leptospirosis and typical gram negative sepsis share similar pathogenesis with regard to systemic endothelial activation; ${ }^{3}$ severe forms of both conditions share the same hemodynamic changes; ${ }^{13}$ and acute respiratory distress syndrome is a common feature of both, ${ }^{3}$ and a recent study suggested that severe pulmonary 
hemorrhagic syndrome in hamsters is parallel with higher local expression of pro-inflammatory genes. ${ }^{14}$

Thalidomide is a well-known immunomodulatory drug that has been widely tested as a possible alternative for treatment of auto-immune diseases and cancer due to its inhibitory effects on TNF- $\alpha$ production and angiogenesis. ${ }^{15}$ In particular, pretreatment with thalidomide prolonged survival in the rat model of sepsis caused by multidrug-resistant Pseudomonas aeruginosa. ${ }^{16}$ This is the only work which evaluates the effect of thalidomide on survival in an experimental model of sepsis. In a previous work, the same group showed that pre-treatment with thalidomide prevented TNF- $\alpha$ elevation in the bloodstream of rats in an experimental model of sepsis by Escherichia coli. In addition, thalidomide prevented secretion of TNF- $\alpha$ by human monocytes after exposure to E. coli. ${ }^{17}$ In Brazil, thalidomide use is licensed, and the drug is distributed only through specific programs of the Ministry of Health to treat the following diseases: hanseniasis-related erythema nodosum, AIDS-related oral aphthous ulcers, multiple myeloma, systemic lupus erythematosus, and Graft-versus-host disease. ${ }^{18,19}$

In previous studies, we developed a model of late antimicrobial treatment in the hamster model of leptospirosis to test adjuvant therapies. ${ }^{6,7}$ In this model, late start of ampicillin results in suboptimal therapy with expected survival rates around $50 \%$. This experimental setting tries to reproduce the common clinical practice in which patients are usually diagnosed late in the course of disease and antibiotics are started four or more days after onset of symptoms. Late antibiotic treatment is the major cause of persistent high case fatality rates in leptospirosis and, as a consequence, adjuvant therapies are urgently needed. The aim of this study was to test whether thalidomide affected survival and target organ pathology when used as an adjuvant therapy in the late introduction of antibiotic therapy hamster model of severe disease. We also investigated if immunomodulatory treatment with thalidomide affected leptospiral load in tissues.

\section{Methods}

\section{Bacteria}

Leptospires were cultivated in liquid Ellinghausen-McCulloughJohnson-Harris (EMJH) medium (Difco Laboratories, Detroit, MI, USA) at $29^{\circ} \mathrm{C}$ and counted in a Petroff-Hausser counting chamber (Fisher Scientific, Pittsburgh, PA, USA). A virulent clinical isolate from Brazil, the Leptospira interrogans serovar Copenhageni strain Fiocruz L1-130, was used in all experiments. ${ }^{20}$ This strain was passaged four times in hamsters and stored at $-70^{\circ} \mathrm{C}$. The aliquots were thawed, and prior to use, they were passaged in liquid medium less than 15 times for a low-passage isolate in the infection experiments. The virulence of this strain was evaluated in hamsters as described previously, and the $50 \%$ lethal dose (LD50) was calculated to be 164 leptospires

\section{Animals and study design}

Nine-week-old female Golden Syrian hamsters (Mesocricetus auratus) (Fiocruz/BA), weighing 60-100 g, were used in all experiments. All hamsters were inoculated intraperitoneally with $10^{6}$ leptospires in $1 \mathrm{ml}$ EMJH medium. All animals were monitored daily for the presence of clinical signs, including alopecia, prostration and photophobia. Ampicillin was administered intramuscularly at a dose of $100 \mathrm{mg} / \mathrm{kg} / \mathrm{bid}$. Treatment started on the selected date and lasted for six days. Thalidomide was supplied as capsules for oral administration. Thalidomide was minced and diluted in commercial linseed oil and administered via a gastric tube at a dose of $50 \mathrm{mg} / \mathrm{kg} .{ }^{16}$ The respective dose of linseed oil administered was $2 \mathrm{ml} / \mathrm{kg}$. Treatment started on the selected date and lasted for three days.

In the two following independent experiments, hamsters were assigned into the following groups: NONE (no intramuscular treatment and pure linseed oil, $2 \mathrm{ml} / \mathrm{kg}$ via a gastric tube); TAL (no intramuscular treatment and thalidomide, $50 \mathrm{mg} / \mathrm{kg}$ diluted in linseed oil, $2 \mathrm{ml} / \mathrm{kg}$ via a gastric tube); AMP (intramuscular ampicillin, $100 \mathrm{mg} / \mathrm{kg} / \mathrm{bid}$ and pure linseed oil, $2 \mathrm{ml} / \mathrm{kg}$ via a gastric tube); and AMP-TAL (intramuscular ampicillin, $100 \mathrm{mg} / \mathrm{kg} / \mathrm{bid}$ and thalidomide, $50 \mathrm{mg} / \mathrm{kg}$ diluted in linseed oil, $2 \mathrm{ml} / \mathrm{kg}$ via a gastric tube). In the first experiment, the start of the treatment was arbitrarily defined as $48 \mathrm{~h}$ after detection of clinical signs in most infected animals. Clinical signs included alopecia, prostration and photophobia. In the second experiment, as an alternative strategy to reproduce the late start of treatment schemes, treatments were started immediately after detection of the first death regardless of the assigned group.

Animals were euthanized in a carbon dioxide chamber when they appeared moribund or at the 28th day after infection (defined as survivors). Necropsies were performed immediately upon euthanasia, and kidney, lung and liver samples were fixed in $4 \%$ formalin, embedded in paraffin and cut into $4-5 \mu \mathrm{m}$ sections for conventional histology.

\section{Outcomes}

The primary outcome was survival. Secondary outcomes that were investigated were: macroscopic pulmonary hemorrhages, tubulointerstitial nephritis, liver lesions and leptospiral load in tissues. At necropsy, the lungs were examined to detect macroscopic pulmonary hemorrhage (PH). Only macroscopic hemorrhages were reported as PH for the purposes of analysis. In all cases, microscopic examination was performed to confirm the presence of massive alveolar hemorrhaging.

\section{Histology}

One kidney of each animal was fixed in $4 \%$ formalin, embedded in paraffin and 4-5 $\mu \mathrm{m}$ thick sections were used for conventional histology. A semi-quantitative estimation of interstitial nephritis was used as previously described. ${ }^{21}$ Briefly, grade + nephritis: infiltrate was rich in macrophages and lymphocytes were restricted to periarterial areas; grade ++ nephritis: infiltrate extended to other renal parenchymal zones with $1-2$ lesions per field of view at $100 x$ magnification; and grade +++ nephritis: lesions detected in more than 2 areas per field of view at 100x magnification. Acute tubular damage (tubular cell swelling), tubular regeneration, glomerular hemorrhage and loss of cohesion (liver-plate disarray) of liver cells were also assessed semi-quantitatively. To test whether immunomodulatory treatment with thalidomide affected leptospiral burden in target organs, we used immunofluorescencebased leptospiral detection and quantification in imprint samples of kidney, liver and lungs as previously described. ${ }^{21-24}$ Leptospiral 
load was expressed as the total count of spiral-shaped organisms in ten fields of view at 400x magnification.

\section{Statistical analysis}

Statistical analyses were performed using the GraphPad Prism 4.03 software package (Graph Pad, La Jolla, CA, USA). Categorical data were compared by Fisher's exact test, and numerical data were compared using a non-parametric MannWhitney test when analyzing differences between two groups. Categorical data were compared by Fisher's exact test, and numerical data were compared using a non-parametric Kruskal-Wallis test when analyzing differences between more than two groups.

Kaplan-Meier curves were established to compare the effects of thalidomide on survival. Curves were compared using log-rank (Mantel-Cox) test. A p value $<0.05$ was considered significant.

\section{Results}

In the first experiment, lethal outcome occurred in $6 / 6$ and $8 / 8$ of the NONE and TAL groups, respectively. No deaths were observed

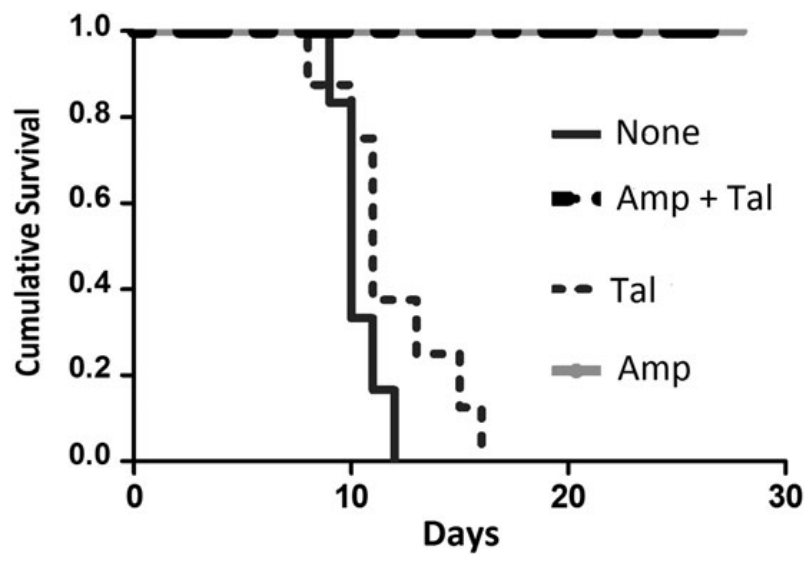

Figure 1. Survival curves of infected hamsters $\left(10^{6}\right.$ Leptospira interrogans strain L1130) treated with ampicillin and/or thalidomide starting $48 \mathrm{~h}$ after the detection of clinical signs (experiment 1). Amp: ampicillin only; Amp-Tal: ampicillin and thalidomide; None: no treatment; Tal: thalidomide only. in animals assigned in the AMP and AMP-TAL groups. Median (25th to 75th percentile) days to death were 10 (10-10.8) and 11 (10.8-13.5) in the NONE and TAL groups, respectively. Survival curves are shown in Figure 1. The differences between curves were significant (log-rank Mantel-Cox test, $p<0.0001$ ). A direct comparison between curves of the NONE and TAL groups showed no difference ( $p>0.05$ ).

The frequency of pulmonary hemorrhages detectable at gross examination was $6 / 6$ (100\%) and 5/8 (63\%) in the NONE and TAL groups. The difference in the frequencies in both groups was significant (Fisher's exact test, $p<0.05$ ). None of the animals defined as survivors (examined at necropsy on day 28) from the AMP and AMP-TAL groups showed macroscopic pulmonary hemorrhages. Table 1 also shows target organ pathology in kidneys and liver at the microscopic level. Animals dying of acute leptospirosis (NONE and TAL groups) showed acute tubular damage (tubular cell swelling), interstitial nephritis, regenerative tubular epithelium and glomerular hemorrhage, as well as liver plate disarray. These lesions were not detected in the AMP and AMP-TAL groups. Frequencies of acute tubular damage and liver plate disarray showed significant differences between groups $(p<0.05)$, while frequencies of tubular regeneration, glomerular hemorrhages and interstitial nephritis were not different ( $p>0.05)$. In contrast, none of the lesions showed significant differences in the frequencies between the NONE and TAL groups. Images of target organ pathology, immunofluorescence-based leptospiral detection and quantification in imprint samples from experimental studies have been extensively illustrated in previous reports. ${ }^{21-25}$

We also investigated the leptospiral load in target organs to determine the extent that immunomodulation by thalidomide could affect the infection burden. Leptospires were detected in kidney samples of $6 / 6$ (100\%), 8/8 (100\%), $1 / 8$ (13\%) and 0/8 hamsters from the NONE, TAL, AMP and AMP-TAL groups, respectively. The leptospiral count was 28 (3-881), 13 (2-131), 0 (0-0) and $0(0-0)$ in the NONE, TAL, AMP and AMP-TAL groups, respectively. Significant differences were observed between groups but not between the NONE and TAL groups. In liver samples, leptospires were detected in 5/6 (83\%), 2/8 (25\%), 0/8 and 0/8 hamsters from the NONE, TAL, AMP and AMP-TAL groups, respectively. The leptospiral count was 13 (1-700), 0 (0-2), $0(0-0)$ and $0(0-0)$ in the NONE, TAL, AMP and AMP-TAL groups, respectively. Significant differences were observed between groups but not between the NONE and TAL groups. Leptospires were detected in the lung samples of $4 / 6(67 \%), 5 / 8(63 \%), 0 / 8$ and $0 / 8$ hamsters from the

Table 1. Target organ pathology in infected hamsters $\left(10^{6}\right.$ Leptospira interrogans strain L1130) treated with ampicillin and/or thalidomide starting $48 \mathrm{~h}$ after the detection of clinical signs (experiment 1 )

\begin{tabular}{lllllll} 
& \multicolumn{2}{l}{ Kidney $\mathrm{n}(\%)$} & & Liver $\mathrm{n}(\%)$ & Lungs $\mathrm{n}(\%)$ \\
\cline { 2 - 7 } & $\begin{array}{l}\text { Tubular cell } \\
\text { swelling }\end{array}$ & $\begin{array}{l}\text { Interstitial } \\
\text { nephritis }\end{array}$ & $\begin{array}{l}\text { Tubular } \\
\text { regeneration }\end{array}$ & $\begin{array}{l}\text { Glomerular } \\
\text { hemorrhage }\end{array}$ & $\begin{array}{l}\text { Liver plate disarray } \\
\text { Macroscopy pulmonary } \\
\text { hemorrhage }\end{array}$ \\
\hline No treatment & $4 / 5(80)$ & $0 / 5$ & $1 / 5(20)$ & $3 / 5(60)$ & $4 / 5(80)$ & $3 / 5(60)$ \\
Thalidomide only & $4 / 7(57)$ & $1 / 7(14)$ & $2 / 7(29)$ & $3 / 7(43)$ & $3 / 7(43)$ & $4 / 7(57)$ \\
Ampicillin only & $0 / 8$ & $0 / 8$ & $0 / 8$ & $0 / 8$ & $0 / 8$ & $0 / 8$ \\
Ampicillin and thalidomide & $0 / 8$ & $0 / 8$ & $0 / 8$ & $0 / 8$ & $0 / 8$ & $0 / 8$
\end{tabular}


NONE, TAL, AMP and AMP-TAL groups, respectively. The leptospiral count was 6 (0-200), 1 (1-11), 0 (0-0) and 0 (0-0) in the NONE, TAL, AMP and AMP-TAL groups, respectively. Significant differences were observed between groups but not between the NONE and TAL groups.

In the second experiment, lethal outcome occurred in $6 / 6$ (100\%), 8/8 (100\%), 6/8 (75\%) and 6/8 (75\%) of the NONE, TAL, AMP and AMP-TAL groups, respectively. The median (25th to 75th percentile) days to death was 6.5 (6-7.8), 9 (8-9.3), 9 (9-9.8) and 9.5 (8.3-10) for the NONE, TAL, AMP and AMP-TAL groups, respectively. Survival curves are shown in Figure 2. The differences between curves were significant (log-rank Mantel-Cox test, $p<0.0001)$. A direct comparison between the curves of the NONE and TAL groups showed significant differences $(p<0.05)$.

Pulmonary hemorrhages were detected in hamsters which succumbed to acute disease. The difference of frequencies between groups was not significant (Fisheŕs exact test, $p>0.05$ ). Table 2 also shows target organ pathology in kidneys and liver at the microscopic level. Frequencies of acute tubular damage and glomerular hemorrhages in animals that died of acute disease were not different between groups. The frequency of tubular

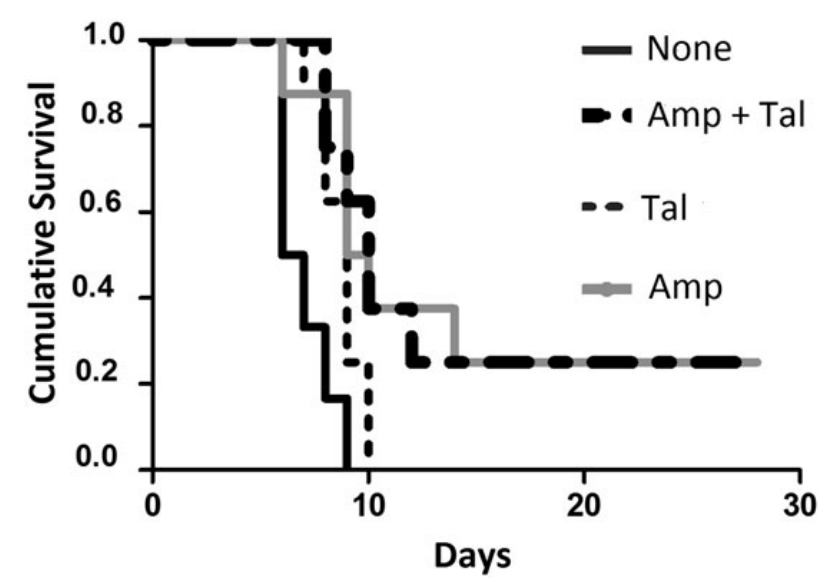

Figure 2. Survival curves of infected hamsters $\left(10^{6}\right.$ Leptospira interrogans strain L1130) treated with ampicillin and/or thalidomide starting immediately after detection of the first death (experiment 2). Amp: ampicillin only; Amp-Tal: ampicillin and thalidomide; None: no treatment; Tal: thalidomide only. regeneration was higher in the TAL, AMP and AMP-TAL groups, although the difference was not significant ( $p>0.05$ ). Liver plate disarray was more common in the NONE group $(p<0.05)$. Survivors exhibited no lesions in kidney and liver samples.

In experiment 2, the leptospiral load was significantly lower in treated groups, including the TAL group. Leptospires were detected in kidney samples of 6/6 (100\%), 4/8 (50\%), 5/8 (63\%) and $2 / 8$ (25\%) hamsters from the NONE, TAL, AMP and AMP-TAL groups, respectively. The leptospiral count was 234 (13-1250), 1 (0-5), $2(0-20)$ and $0(0-1)$ in the NONE, TAL, AMP and AMP-TAL groups, respectively. Differences were significant between the groups $(p<0.05)$ and also between the NONE and TAL groups $(p<0.05)$. Leptospires were detected in liver samples of 5/6 (83\%), $2 / 8(25 \%), 3 / 8(38 \%)$ and $0 / 8$ hamsters from the NONE, TAL, AMP and AMP-TAL groups, respectively. The leptospiral count was 14 (4-1213), $0(0-1), 0(0-2)$ and $0(0-0)$ in the NONE, TAL, AMP and AMP-TAL groups, respectively. Differences were significant between groups $(p<0.05)$ and also between the NONE and TAL groups $(p<0.05)$. Leptospires were detected in lung samples of $4 / 6(67 \%), 0 / 8,1 / 8(13 \%)$ and $0 / 8$ hamsters from the NONE, TAL, AMP and AMP-TAL groups, respectively. The leptospiral count was 11 (0-311), $0(0-0), 0(0-0)$ and $0(0-0)$ in the NONE, TAL, AMP and AMP-TAL groups, respectively. Differences were significant between the groups $(p<0.05)$ and also between the NONE and TAL groups $(p<0.05)$.

\section{Discussion}

Handling leptospires in vitro and dealing with experimental animal models of leptospirosis is a challenging endeavor. Minimal variations on in vitro passages, defrosting procedures and in animal conditions may result in significant changes in bacterial virulence and the outcome of experimental infections. In this context, it is difficult to establish a model for the late start of antibiotic therapy because more variables are added to this model, which is already known to show some inherent variability. While some innate difficulties of the late start model are clear, we believe that there is no other strategy to test urgently needed adjuvant therapies for leptospirosis under experimental conditions. The strategies presented herein aim to reproduce the common clinical setting in which patients with severe leptospirosis seek medical assistance after more than four days of symptoms: usually a

Table 2. Target organ pathology in infected hamsters $\left(10^{6}\right.$ Leptospira interrogans strain L1130) treated with ampicillin and/or thalidomide starting immediately after detection of the first death (experiment 2)

\begin{tabular}{lllllll} 
& \multicolumn{2}{l}{ Kidney $\mathrm{n}(\%)$} & & Liver $\mathrm{n}(\%)$ & Lungs $\mathrm{n}(\%)$ \\
\cline { 2 - 7 } & $\begin{array}{l}\text { Tubular cell } \\
\text { swelling }\end{array}$ & $\begin{array}{l}\text { Interstitial } \\
\text { nephritis }\end{array}$ & $\begin{array}{l}\text { Tubular } \\
\text { regeneration }\end{array}$ & $\begin{array}{l}\text { Glomerular } \\
\text { hemorrhage }\end{array}$ & $\begin{array}{l}\text { Liver plate disarray } \\
\text { Macroscopy pulmonary } \\
\text { hemorrhage }\end{array}$ \\
\hline No treatment & $6 / 6(100)$ & $0 / 6$ & 0 & $1 / 6(17)$ & $5 / 6(83)$ & $1 / 6(17)$ \\
Thalidomide only & $5 / 8(63)$ & $0 / 8$ & $3 / 8(38)$ & $1 / 8(13)$ & $0 / 8$ & $1 / 8(13)$ \\
Ampicillin only & $3 / 8(38)$ & $0 / 8$ & $4 / 8(50)$ & $0 / 8$ & $1 / 8(13)$ & $0 / 8$ \\
Ampicillin and thalidomide & $4 / 8(50)$ & $0 / 8$ & $3 / 8(38)$ & $0 / 8$ & $1 / 8(13)$ & $2 / 8(25)$
\end{tabular}


period of unspecific symptoms that can be easily confounded with dengue fever or a less severe self-resolving febrile illnesses.

The issue of immunomodulatory treatment in experimental leptospirosis has been poorly explored. Yukawa and colleagues tested the effects of combined or isolated therapies with penicillin and subcutaneous steroid therapy in gerbils. ${ }^{26}$ The work did not reproduce the late start of treatment setting. Indeed, treatments were started three, two or one day before infection or at the moment of infection. As a consequence, this scheme reproduces chemoprevention rather than treatment of an established infection. In addition, as expected, gerbils pretreated with steroids had shortened survival. Similarly, in an experimental trial to compare ampicillin, ofloxacin, and doxycycline in hamsters, treatments were arbitrarily started after three days of infection, which was most likely too soon to reproduce clinical disease and the real setting of patients. ${ }^{9}$ Even the work that suggested the benefit on survival of thalidomide in a sepsis model was also based on a pre-treatment with thalidomide thirty minutes before intraperitoneal infection with a multidrug-resistant blood isolate of Pseudomonas aeruginosa. ${ }^{16}$ In that case, however, the disease had a short evolution, with deaths detected between 20 and 180 hours after infection. In the present study, we aimed to check differences on survival in a disease that usually follows a course of 10 to 14 days in lethal cases under experimental conditions. Therefore, the study design of a late start of antibiotic treatment and immunomodulation was imperative.

Five or six days of ampicillin are already standardized treatments for experimental leptospirosis. We arbitrarily defined the duration of thalidomide treatment as three days. As stated above, other studies focusing on immunomodulatory treatment with thalidomide or steroids were based in pre-infection administration of these. Thus, even arbitrarily defined, the study design of using thalidomide mirroring clinical practice is one of the strengths of the present work.

Regarding immunomodulation, we have previously tested $\mathrm{N}$-acetylcysteine ${ }^{7}$ and methylene blue ${ }^{6}$ as potential adjuvants and observed no additional benefit in experimental disease when compared to ampicillin alone. In the former, study design included: treatments starting after detection of clinical signs of disease; and treatments starting six days after infection. In the latter, survival was evaluated in a experiment with the same design of the second experiment from this study: late treatment was reproduced by starting treatments immediately after detection of the first lethal outcome. ${ }^{6}$

It is noteworthy that even in the absence of supportive experimental data, some studies have already been performed on immunomodulation in patients with severe leptospirosis. There are anecdotal reports of clinical benefits of high doses of methyl prednisolone in patients with severe pulmonary hemorrhagic syndrome. ${ }^{27-29}$ A report from Sri Lanka describes the experience after the introduction (in 2008) of bolus methylprednisolone as a standard therapy for severe leptospirosis: lethality dropped from $17 / 78(22 \%)$ to $16 / 148(11 \%) .{ }^{30}$ However, a unique prospective randomized controlled trial on this issue was performed in Thailand: desmopressin or pulse dexamethasone were tested as adjuvant therapies in 68 patients with severe pulmonary hemorrhagic syndrome and showed no benefit. ${ }^{31}$ In addition to their report of clinical benefit of steroid pulse therapy, ${ }^{27}$ Trivedi and colleagues have reported that other immunosuppressive adjuvant treatments, namely cyclophosphamide and plasma exchange with immunosuppression followed by cyclophosphamide, improved survival. ${ }^{32,33}$

In experiment 1 , the analysis of the effect of thalidomide on survival when used in combination with ampicillin therapy was not possible because all animals in the AMP and AMP-TAL groups survived. This was not expected based on preliminary data (Table 1); however, some variation in virulence of strains is common in practice of experimental leptospirosis. A minor loss of virulence in experiment 1 resulted, which indicated that starting treatment at the 10th day was too early to reproduce late therapy. Therefore, high survival (higher than expected) precluded analysis of the effect of combined ampicillin and thalidomide therapy. Although the difference was not significant, hamsters treated with thalidomide only survived longer than untreated animals. Thalidomide did not influence leptospiral load in tissues. Only animals that developed acute lethal disease showed pulmonary hemorrhages, kidney lesions and liver plate disarray. No pathology was detected in survivors.

Experiment 2 successfully reproduced late (suboptimal) ampicillin therapy. The rate of lethal outcome was the same (6/8,75\%) for both the AMP and AMP-TAL groups, and the survival curves were not significantly different. In contrast, hamsters treated with thalidomide only survived longer than untreated animals, and this difference was significant. Because lethal outcomes were observed in all groups, target organ lesions were detected in all groups. The frequency of two lesions, regeneration of tubular epithelium and liver plate disarray were significantly different between groups. More than from a direct effect of treatment, these differences may derive from a longer interval between infection and death. Liver plate disarray is a typical feature of acute-phase disease and may resolve in delayed cases of infection during renal disease progression. Conversely, regenerative epithelium most likely reflects previous acute tubular damage and may not be detected in cases of early lethal outcome. The fact that target organ lesions vary as a function of time (acute vs convalescence phase) is well characterized in humans and experimental leptospirosis. . $^{54}$

Comparing only the NONE group in experiments 1 and 2, the median was 10 (10-10.8) and 6.5 (6-7.8) (log-rank Mantel-Cox test, $p<0.0001$ ), respectively, thus implying that the strain, even if maintained at the same strict conditions, was more virulent in the second experiment. In experiment 1 , symptoms were first detected at day 4 . An overall median (25th to 75 th percentile) day to death (regardless of assigned groups) was 11 (10-11.8). In experiment 2, symptoms were first detected at day 2 , and the first death was observed at day 4. Therefore, treatment was started two days after the onset of symptoms. An overall median (25th to 75th percentile) day to death (regardless of assigned groups) was 9 (8-9.8). Treatments were started two days after appearance of clinical signs in both experiments, however, experiment 2 showed a more rapidly progressing disease. Thus, the variability of virulence and the different study design resulted in later treatment in experiment 2, allowing the occurrence of death in the ampicillin-treated groups. Comparing the outcomes of both experiments, it seems that waiting for the first death to begin therapy is a feasible way to reproduce the late start of treatment under these experimental conditions. Waiting for the first death to occur precludes the potential variability of the best day to begin therapy, as previously defined in other experiments as being due 
to a possible loss of virulence of the strain, and avoids the requirement of time consuming preliminary experiments.

The leptospiral load in experiment 2 was consistently higher in the untreated groups when compared to the treated groups, including the TAL group. Differences in the leptospiral load in kidneys and lungs were significant between the NONE and TAL groups. An obvious conclusion is that short-term immunomodulation by thalidomide does not result in a higher leptospiral burden in tissues. However, this observation is not entirely unexpected. Thalidomide use may provide clinical benefit for patients with some infectious diseases, such as mycobacteriosis and microspordial diarrhea, in clinical patients; however, no direct effect on microorganism load has been demonstrated. ${ }^{35,36}$ An alternative explanation, however, is that any treatment (including immunomodulation only) results in prolonged survival. Therefore, differences in bacterial load may reflect that the natural immune response is to clear leptospires from tissues over time, and thus, animals dying later may show a lower leptospiral concentration in tissues. Truccolo and colleagues have shown that after reaching the threshold of $10^{4}$ leptospires $/ \mathrm{ml}$ in blood, patients eventually succumb to leptospirosis despite aggressive antimicrobial treatment. Lethal outcome occurred even in patients who had reduced leptospiremia on follow-up: thus implying an immune mediated mechanisms associated with clinical complications ${ }^{37}$ As a result, acute leptospirosis may be fatal even if the leptospiral load decreases or is undetected in target organs.

In conclusion, we report a model of a late start of antibiotic therapy in the hamster model of leptospirosis to test adjuvant therapies. In the present study, thalidomide showed a limited effect on outcome, and thus, the present data does not support testing thalidomide as an adjuvant therapy in patients with leptospirosis. We hope that this report will encourage further research on potential adjuvant therapies for leptospirosis.

Authors' contributions: DAA conceived the study; DAA and LMS designed the study protocol; LMS, JOM, ECA, CSS, MQS and ACS carried out the experimental assessment; DAA and LMS drafted the manuscript; MGR critically revised the manuscript for intellectual content. All authors read and approved the final manuscript. LMS and DAA are guarantors of the paper.

Acknowledgements: The authors are grateful to Dr Euzenir Sarno for kindly providing us with thalidomide.

\section{Competing interests: None declared.}

Funding: This work was supported by grants from FAPESB (Bahia State Research Funding Agency), APP0057/2009, and CNPq (National Council for Scientific and Technological Development) 470021/2011-0.

Ethical approval: The Ethical Committee of the Oswaldo Cruz Foundation approved all of the animal experimental protocols used in this study (CEUA 011/2010).

\section{References}

1 Bharti AR, Nally JE, Ricaldi JN et al. Leptospirosis: a zoonotic disease of global importance. Lancet Infect Dis 2003;3:757-71.
2 McBride AJ, Athanazio DA, Reis MG et al. Leptospirosis. Curr Opin Infect Dis 2005;18:376-86.

3 Medeiros FR, Spichler A, Athanazio DA. Leptospirosis-associated disturbances of blood vessels, lungs and hemostasis. Acta Trop 2010;115:155-62.

4 WHO. Human leptospirosis: guidance for diagnosis, surveillance and control. Malta: World Health Organization; 2003.

5 Silva EF, Santos CS, Athanazio DA et al. Characterization of virulence of Leptospira isolates in a hamster model. Vaccine 2008;26:3892-6.

6 Santos CS, de Azevedo EC, Soares LM et al. Ionic imbalance and lack of effect of adjuvant treatment with methylene blue in the hamster model of leptospirosis. Mem Inst Oswaldo Cruz 2013;108:438-45.

7 Spichler A, Ko AI, Silva EF et al. Reversal of renal tubule transporter downregulation during severe leptospirosis with antimicrobial therapy. Am J Trop Med Hyg 2007;77:1111-9.

8 Alexander AD, Rule PL. Penicillins, cephalosporins, and tetracyclines in treatment of hamsters with fatal leptospirosis. Antimicrob Agents Chemother 1986;30:835-9.

9 Truccolo J, Charavay F, Merien F et al. Quantitative PCR assay to evaluate ampicillin, ofloxacin, and doxycycline for treatment of experimental leptospirosis. Antimicrob Agents Chemother 2002;46:848-53.

10 Hotchkiss RS, Karl IE. The pathophysiology and treatment of sepsis. N Engl J Med 2003;348:138-50.

11 Tajiki H, Salomao R. Association of plasma levels of tumor necrosis factor alpha with severity of disease and mortality among patients with leptospirosis. Clin Infect Dis 1996;23:1177-8.

12 Wagenaar JF, Goris MG, Gasem MH et al. Long pentraxin PTX3 is associated with mortality and disease severity in severe Leptospirosis. J Infect 2009;58:425-32.

13 Siriwanij T, Suttinont C, Tantawichien T et al. Haemodynamics in leptospirosis: effects of plasmapheresis and continuous venovenous haemofiltration. Nephrology (Carlton) 2005;10:1-6.

14 Marinho M, Oliveira-Júnior IS, Monteiro CMR et al. Pulmonary disease in hamsters infected with Leptospira interrogans: histopathologic findings and cytokine mRNA expressions Am J Trop Med Hyg 2009;80:832-6.

15 Chen M, Doherty SD, Hsu S. Innovative uses of thalidomide. Dermatol Clin 2010;28:577-86.

16 Giamarellos-Bourboulis EJ, Bolanos N, Laoutaris G et al. Immunomodulatory intervention in sepsis by multidrug-resistant Pseudomonas aeruginosa with thalidomide: an experimental study. BMC Infect Dis 2005;5:51.

17 Giamarellos-Bourboulis EJ, Poulaki H, Kostomitsopoulos $\mathrm{N}$ et al. Effective immunomodulatory treatment of Escherichia coli experimental sepsis with thalidomide. Antimicrob Agents Chemother 2003;47:2445-9.

18 Oliveira MA, Bermudez JAZ, Souza ACM. Talidomida no Brasil: Vigilância com responsabilidade compartilhada? Cad Saude Publica 1999;15:99-112.

19 Schuler-Faccini L, Soares RC, de Sousa AC et al. New cases of thalidomide embryopathy in Brazil. Birth Defects Res A Clin Mol Teratol 2007;79:671-2.

20 Nascimento AL, Ko AI, Martins EA et al. Comparative genomics of two Leptospira interrogans serovars reveals novel insights into physiology and pathogenesis. J Bacteriol 2004;186:2164-72.

21 Bandeira M, Santos CS, de Azevedo EC et al. Attenuated nephritis in inducible nitric oxide synthase knockout C57BL/6 mice and pulmonary hemorrhage in CB17 SCID and recombination activating 
gene 1 knockout C57BL/6 mice infected with Leptospira interrogans. Infect Immun 2011;79:2936-40.

22 Chagas-Junior AD, da Silva CL, Soares LM et al. Detection and quantification of Leptospira interrogans in hamster and rat kidney samples: immunofluorescent imprints versus real-time PCR. PLoS One 2012;7:e32712.

23 Chagas-Junior AD, McBride AJ, Athanazio DA et al. An imprint method for detecting leptospires in the hamster model of vaccine-mediated immunity for leptospirosis. J Med Microbiol 2009;58:1632-7.

24 Santos CS, Macedo JO, Bandeira M et al. Different outcomes of experimental leptospiral infection in mouse strains with distinct genotypes. J Med Microbiol 2010;59:1101-6.

25 Athanazio DA, Santos CS, Santos AC et al. Experimental infection in tumor necrosis factor alpha receptor, interferon gamma and interleukin 4 deficient mice by pathogenic Leptospira interrogans. Acta Trop 2008;105:95-8.

26 Yukawa M, Kamata $\mathrm{H}$, Ohba $\mathrm{S}$ et al. Effect of immune serum, an antibiotic, and a corticosteroid used alone or in combination on experimental leptospirosis in Mongolian gerbils (Meriones unguiculatus). J Basic Microbiol 1994;34:49-55.

27 Trivedi SV, Chavda RK, Wadia PZ et al. The role of glucocorticoid pulse therapy in pulmonary involvement in leptospirosis. J Assoc Physicians India 2001;49:901-3.

28 Thunga G, John J, Sam KG et al. Role of high-dose corticosteroid for the treatment of leptospirosis-induced pulmonary hemorrhage. J Clin Pharmacol 2012;52:114-6.
29 Courtin JP, Carre P, Poubeau P et al. Diffuse alveolar hemorrhage and myositis in icterohemorrhagic leptospirosis. Rapid control by a single bolus of corticoids [in French]. Rev Mal Respir 1994;11:601-3.

30 Kularatne SA, Budagoda BD, de Alwis VK et al. High efficacy of bolus methylprednisolone in severe leptospirosis: a descriptive study in Sri Lanka. Postgrad Med J 2011;87:13-7.

31 Niwattayakul K, Kaewtasi S, Chueasuwanchai S et al. An open randomized controlled trial of desmopressin and pulse dexamethasone as adjunct therapy in patients with pulmonary involvement associated with severe leptospirosis. Clin Microbiol Infect 2010;16:1207-12.

32 Trivedi SV, Vasava AH, Bhatia LC et al. Plasma exchange with immunosuppression in pulmonary alveolar haemorrhage due to leptospirosis. Indian J Med Res 2010;131:429-33.

33 Trivedi SV, Vasava AH, Patel TC et al. Cyclophosphamide in pulmonary alveolar hemorrhage due to leptospirosis. Indian J Crit Care Med 2009;13:79-84

34 Arean VM. The pathologic anatomy and pathogenesis of fatal human leptospirosis (Weil's disease). Am J Pathol 1962;40:393-423.

35 Ridoux O, Drancourt M. Lack of in vitro antimicrosporidian activity of thalidomide. Antimicrob Agents Chemother 1999;43:2305-6.

36 Tadesse A, Shannon EJ. Effects of thalidomide on intracellular Mycobacterium leprae in normal and activated macrophages. Clin Diagn Lab Immunol 2005;12:130-4.

37 Truccolo J, Serais O, Merien F et al. Following the course of human leptospirosis: Evidence of a critical threshold for the vital prognosis using a quantitative PCR assay. FEMS Microbiology Letters 2001;204:317-21. 